\title{
EVALUACIÓN DE IMPLEMENTOS DE LABRANZA A DIFERENTES VELOCIDADES DE OPERACIÓN Y CONTENIDOS DE AGUA DEL SUELO
}

\section{Evaluation of tillage tools at different working speeds and soil water contents}

\author{
Jesús H. Camacho-Tamayo ${ }^{1}$ *, Gonzalo A. Rodríguez B. ${ }^{2}$
}

\begin{abstract}
A B S T R A C T
Soil tillage is a relevant agricultural practice with important effects on soil physical properties and energy use. The objective of this study was to measure the cross-sectional area of worked soil, working depth, working width, draught and specific draught for different tillage implements, including disc plow, vibratory chisel plow and rigid chisel plow, at three soil water contents $\left(0.05 ; 0.12\right.$ y $\left.0.15 \mathrm{~g} \mathrm{~g}^{-1}\right)$ and three forward speeds $\left(1.12 ; 1.45\right.$ and $\left.1.80 \mathrm{~m} \mathrm{~s}^{-1}\right)$. The results showed that water content affects all the parameters analyzed, particularly for disk and vibratory chisel plow. The rigid chisel plow is energy efficient to loosen soil, with low specific draught and high crosssectional area of working soil. Tillage at $0.12 \mathrm{~g} \mathrm{~g}^{-1}$ water content gave the lowest draught requirement for soil aggregate reduction.
\end{abstract}

Key words: chisel plow, disc plow, specific draught, agricultural machinery.

\section{R E S U M E N}

La labranza del suelo es de gran importancia puesto que altera las propiedades físicas y representa un alto consumo de energía. El objetivo del presente estudio fue medir el área transversal del suelo disturbado, la profundidad de trabajo, el ancho de trabajo, la fuerza de tracción y la resistencia específica para diferentes implementos de labranza, arado de discos, arado de cincel vibratorio y arado de cincel rígido, con tres contenidos de agua $\left(0,05 ; 0,12\right.$ y $\left.0,15 \mathrm{~g} \mathrm{~g}^{-1}\right)$ y tres velocidades de operación $\left(1,12 ; 1,45\right.$ y $\left.1,80 \mathrm{~m} \mathrm{~s}^{-1}\right)$. El contenido de agua afectó todos los parámetros analizados, especialmente para el arado de discos y el arado de cincel vibratorio. Los resultados muestran que el arado de cincel rígido es energéticamente más eficiente para preparar el suelo, con una resistencia específica baja y una mayor área transversal de suelo disturbado. La preparación del suelo a un $0,12 \mathrm{~g} \mathrm{~g}^{-1}$ de contenido en agua, presentó la resistencia específica más baja para la fragmentación del suelo.

Palabras clave: arado de cincel, arado de discos, resistencia específica, maquinaria agrícola.

\footnotetext{
${ }^{1}$ Universidad Nacional de Colombia, Sede Bogotá, Facultad de Ingeniería, Programa de Ingeniería Agrícola, Ciudad Universitaria, Bogotá, Colombia. E-mail: jhcamachot@unal.edu.co *Autor para correspondencia.

${ }^{2}$ Corporación Colombiana de Investigación Agropecuaria, Centro de Investigación Tibaitatá, km 14 Vía Mosquera, Bogotá, Colombia. E-mail: grodriguez@corpoica.org.co

Recibido: 4 de julio de 2006. Aceptado: 20 de octubre de 2006.
} 


\section{INTRODUCCIÓN}

La labranza primaria adquiere importancia en la preparación del suelo debido a que la remoción es alta y que esta labor afecta en gran medida sus propiedades físicas, y directa e indirectamente las propiedades químicas y biológicas. Estos cambios deben favorecer la producción de los cultivos y mantener la calidad del suelo, pero debido al uso inadecuado de los implementos de labranza se provoca la degradación de éste. La búsqueda de soluciones a esta problemática ha conducido al estudio e implantación de la labranza de conservación, con el fin de disminuir el impacto sobre el medio ambiente y especialmente sobre el suelo. Por lo anterior, es importante conocer diferentes parámetros operativos de los implementos de labranza y su incidencia en el suelo.

De acuerdo con Aluko y Seig (2000) la movilización del suelo es causada por cizallamiento, donde las partículas se someten a compresión por tensión, situación en que el suelo se agrieta, y por deformación plástica, situación indeseada, ya que el suelo sólo se deforma en la superficie de contacto con la herramienta, lo cual puede conllevar a la compactación.

Para cinceles y subsoladores es importante considerar el ángulo de ataque, ya que para un valor cercano 0,436 rad se presentan bajos valores de fuerza de tracción (Aluko y Seig, 2000), así como la menor resistencia específica (Magalhães y Souza, 1990; Chaudhuri, 2001). Por otra parte, McKyes (1985) describe que para cinceles existe una profundidad de trabajo crítica, en la cual no existe remoción lateral de suelo, y que ésta depende del ancho del implemento y de su ángulo de ataque, así como de la densidad y contenido de humedad del suelo. Para trabajar en profundidades mayores a la crítica, es recomendable el uso de aletas laterales en la punta del cincel, lo cual reduce también la resistencia específica y favorece el ancho de trabajo y la eficiencia de campo (McKyes, 1985).

Para los arados de discos, Godwin et al. (1987) encontraron que la fuerza de tracción es mínima donde el ángulo de inclinación vertical está entre 0,380 y $0,524 \mathrm{rad}$, siendo la resistencia específica mínima para un ángulo de $0,436 \mathrm{rad}$, coincidiendo con Gill et al. (1982), quienes también encontraron que la penetración de los discos es mayor si se aumenta el peso de éstos, y que la velocidad de operación no afecta la profundidad de trabajo en suelos con altos contenidos de arena, pero en suelos arcillosos ésta disminuye a medida que se aumenta la velocidad de operación. Por otra parte, Mello y Magalhães (1995) en un trabajo realizado sobre un suelo arcilloso, encontraron que la velocidad de operación no afectó la fuerza de tracción, y que dicha fuerza crece al aumentar el ángulo horizontal y el área de suelo removido, sin incidir significativamente en la resistencia específica.

La humedad del suelo es un factor que incide directamente en la fuerza de tracción y requerimiento de potencia en la preparación del suelo. Suelos secos presentan agregados con alta cohesión, siendo más alta en suelos arcillosos, requiriendo mayor fuerza de tracción para ser disturbados. La cohesión disminuye a medida que el contenido de agua aumenta (Mouazen y Ramón, 2002), aumentando entonces la adhesión de las partículas del suelo sobre la superficie de la herramienta y afectando la fuerza de tracción. Arvidsson et al. (2004) encontraron que el contenido de agua adecuado para las labores de labranza es cercano al límite plástico del suelo; también concluyeron que la profundidad de trabajo aumenta a mayor contenido de humedad del suelo, disminuyendo la resistencia específica. Por otra parte, la labor en un suelo seco favorece la formación de terrones grandes (De Toro y Arvidsson, 2003) y suelos con contenido de agua superior al límite plástico son fácilmente deformados y compactados.

Con el fin de establecer las condiciones del suelo adecuadas para laboreo, reducir el impacto sobre el medio ambiente, buscando un bajo consumo de potencia, el objetivo del presente trabajo fue analizar la remoción del suelo, la fuerza de tracción necesaria para la operación de tres tipos de implementos de labranza primaria, en un suelo con tres contenidos de agua y tres velocidades de operación.

\section{MATERIALES Y MÉTODOS}

Las pruebas se realizaron en la Estación Experimental Taluma, de la Corporación Colombiana de Investigación Agropecuaria (CORPOICA), ubicada en el municipio de Puerto López (Meta, Colombia), entre las coordenadas mínimas 1’204.377 E y 973.655 N, máximas 1'206.824 E y 976.874 N y 300 m.s.n.m. El suelo es un oxisol (Typic Haplus- 
tox) de textura franca y contenidos medios de $22,1 \%$ de arcilla, $40,5 \%$ de limo y $37,4 \%$ de arena, con densidad aparente de $1.430 \mathrm{~kg} \mathrm{~m}^{-3} \mathrm{y}$ densidad real de $2.450 \mathrm{~kg} \mathrm{~m}^{-3}$. Los ensayos de campo se realizaron utilizando elementos individuales del arado de discos, arado de cincel vibratorio y arado de cincel rígido, todos de fabricación colombiana y cuyas características se observan en el Cuadro 1. Antes de realizar las pruebas en campo, se estableció el índice de cono (IC) con el fin de determinar la resistencia a la penetración del suelo en cada lote de ensayo, para posteriormente determinar el área de suelo disturbada (Ad), el ancho de trabajo (At), la profundidad de trabajo $(\mathrm{Pt})$, considerados de acuerdo con la Figura 1 y determinados mediante un perfilómetro de 30 varillas, distanciadas $50 \mathrm{~mm}$ entre una y otra, similar al utilizado por Camacho y Magalhães (2004).
Para el trabajo en el campo, el operario iba introduciendo la unidad del arado evaluada hasta la profundidad donde las condiciones del suelo o las características del implemento lo permitían. Usando un dinamómetro mecánico precisión del 0,5\% (Dillon, modelo AP, Fairmont, Minnesota, USA), se estableció la fuerza de tracción (F), para luego calcular la resistencia específica $\left(\mathrm{R}_{\mathrm{E}}=\mathrm{F}^{*} \mathrm{Ad}^{-1}\right)$. Las velocidades de operación fueron de 1,$12 ; 1,45 \mathrm{y}$ $1,80 \mathrm{~m} \mathrm{~s}^{-1}$, referidas como V1, V2 y V3, respectivamente, y los contenidos de agua del suelo fueron de 0,$05 ; 0,12$ y $0,15 \mathrm{~g} \mathrm{~g}^{-1}$, referidos como H1, H2 y $\mathrm{H} 3$, respectivamente. El tractor utilizado para las pruebas de campo disponía de una potencia en el motor de $59,6 \mathrm{~kW}$.

El diseño experimental fue de bloques al azar, realizando cuatro repeticiones por tratamiento y tres

Cuadro 1. Especificaciones de las unidades de arado usadas.

Table 1. Main specifications of the tillage tools used.

\begin{tabular}{lll}
\hline Unidad de arado evaluada & Características \\
\hline Disco & $660 \mathrm{~mm}$ de diámetro \\
- Ángulo de inclinación vertical de $0,384 \mathrm{rad}$ & - Ángulo de inclinación lateral de $0,785 \mathrm{rad}$ \\
- Marca Apolo, Medellín, Colombia
\end{tabular}


Figura 1. Área típica de suelo disturbado con arado de cincel (izquierda) y arado de discos (derecha). Ad = área disturbada; $\mathbf{A t}=$ ancho de trabajo; $\mathbf{P t}=$ profundidad de trabajo.

Figure 1. Typical soil disturbance with chisel plow (left) and disk plow (right). Ad = soil disturbance; At = working width; $\mathbf{P t}=$ working depth.

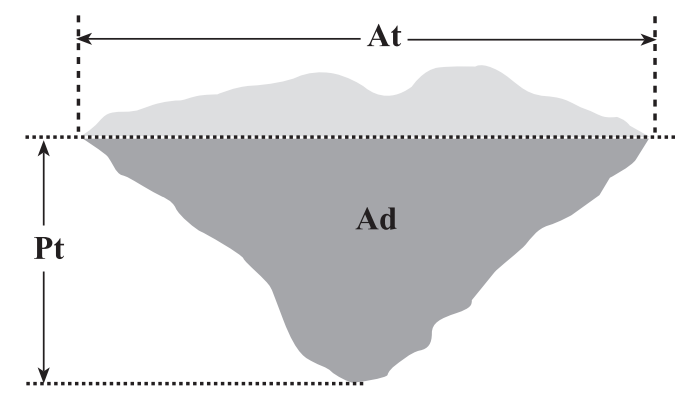

lecturas por repetición, para un total de 324 muestras analizadas, procesadas en el paquete estadístico SPSS (SPSS, 2004). Los datos obtenidos para los parámetros estudiados en los tratamientos fueron analizados mediante la prueba de Duncan $(P<0,05)$.

\section{RESULTADOS Y DISCUSIÓN}

Fuerza de tracción. El contenido de agua del suelo afectó directamente la fuerza de tracción nece-

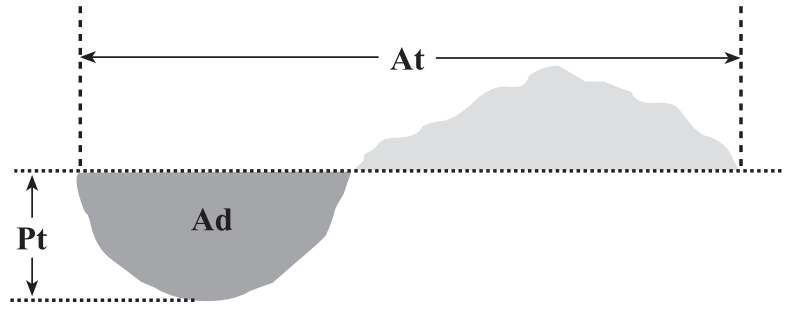

saria para cada implemento, como se observa en la Figura 2, donde el contenido de agua $\mathrm{H} 2$ resulta ser el más adecuado para el laboreo del suelo. Para los cinceles vibratorio y rígido se observó que para H1 (alta cohesión) y H3 (alta adhesión), la fuerza de tracción es alta, efectos también reportados por Mouazen y Ramón (2002) y Arvidsson et al. (2004). Para la unidad del arado de discos se observó que la fuerza de tracción presenta una relación directa con el contenido de agua, debido a que la adhesión crece, pues la superficie de contacto entre el suelo

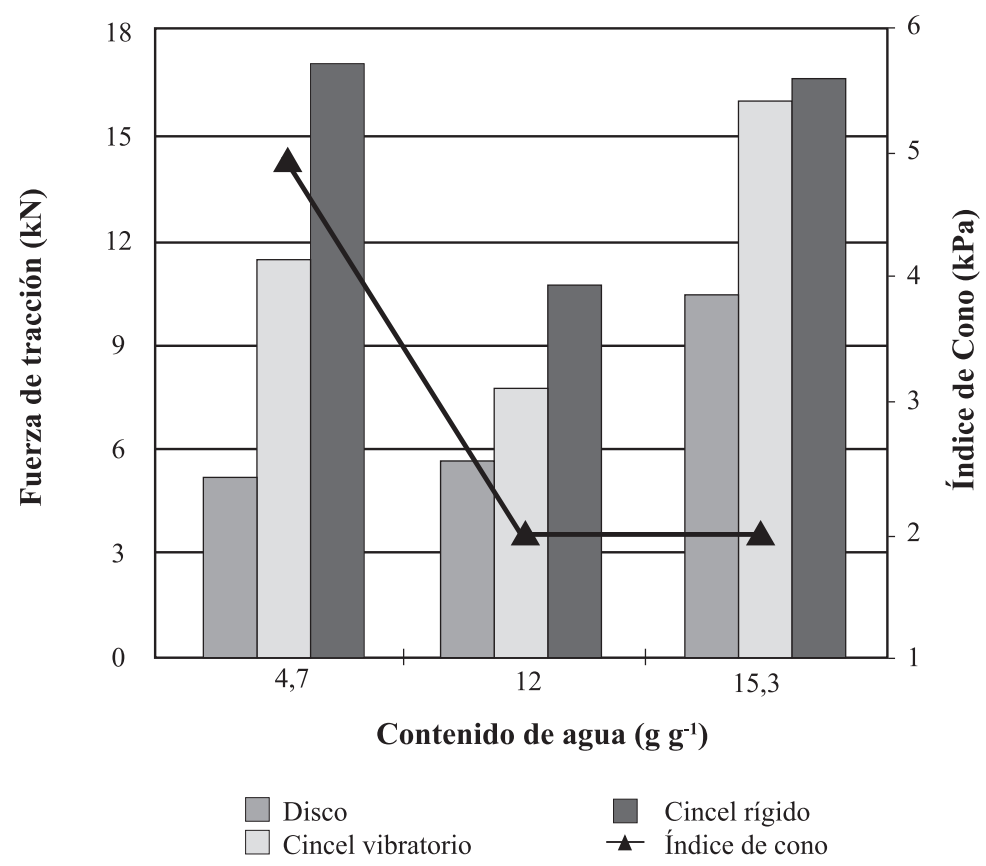

Figura 2. Fuerza de tracción (F) e índice de cono (IC) para tres implementos de labranza y diferentes contenidos de agua del suelo.

Figure 2. Draught force (F) and cone index (IC) for three tillage tools at different soil water contents. 
y la herramienta es mayor en el caso de los discos. La diferencia de la fuerza de tracción requerida por los implementos en estudio, igualmente se explica por la geometría de éstos y por la diferencia presentada en la profundidad de trabajo. También se observó que el índice de cono disminuye con el contenido de agua y no presentó una relación directa con la fuerza de tracción.

Área de suelo disturbada. La menor área disturbada se presentó con el cincel vibratorio y el disco, los cuales no difirieron estadísticamente para un mismo contenido de agua del suelo, pero al comparar estos implementos con el cincel rígido, se observó que son estadísticamente diferentes y que el cincel rígido presenta la mayor área disturbada para todas las condiciones de contenido de agua del suelo y velocidades de operación evaluadas (Cuadro 2). Por otra parte, el contenido de agua afecta este parámetro, observándose que $\mathrm{H} 2$ es el contenido de agua más adecuado para laborar el suelo, tanto en el caso del disco como en el del cincel vibratorio. En el caso del cincel rígido, se observó que la mayor área disturbada se presenta con el contenido de agua $\mathrm{H} 1$, con formación de terrones de gran tamaño, condición no deseable para posteriores labores de desterronamiento y siembra, siendo entonces conveniente realizar esta labor a mayores contenidos de humedad. Además, se observó que las velocidades de operación analizadas no afec- tan significativamente el área de suelo disturbada, corroborando la situación informada por Lanças y Benez (1988) y Coelho (1998).

Profundidad de trabajo. La menor profundidad de trabajo se presentó en el arado de disco, seguido del cincel vibratorio y del cincel rígido (Cuadro 3). El contenido de agua del suelo afectó también la profundidad de trabajo, encontrándose que en el caso del arado de cincel vibratorio este parámetro crece con la humedad, presentando poca profundidad de trabajo con bajos contenidos de agua del suelo. Lo mismo ocurre en el caso del disco, siendo éste el implemento más sensible al contenido de humedad, lo cual coincide con lo reportado por Arvidsson et al. (2004). El arado de cincel rígido fue el implemento que presentó una mayor uniformidad en la profundidad de trabajo, con diferencias significativas respecto a un contenido de agua de H3. Por otra parte, la velocidad de operación no presentó una gran incidencia en la profundidad de trabajo, notándose que ésta disminuye a una mayor velocidad de operación, sin presentar diferencias significativas.

Ancho de trabajo. El cincel rígido presentó el mayor ancho de trabajo en todos los tratamientos estudiados (Cuadro 4), con diferencias significativas respecto a los otros implementos, para todos los contenidos de agua, donde el menor ancho de trabajo se

Cuadro 2. Áreas de suelo disturbadas $\left(\mathrm{m}^{2}\right)$ por los implementos, para diferentes contenidos de agua y velocidades de operación.

Table 2. Disturbed soil area $\left(\mathrm{m}^{2}\right)$ by tillage tools, for different soil water contents and field speeds.

\begin{tabular}{|c|c|c|c|c|c|c|c|c|c|c|}
\hline \multirow{2}{*}{ Implemento } & \multirow{2}{*}{ Humedad } & \multicolumn{7}{|c|}{ Velocidad de operación } & \multirow{2}{*}{ Media } & \multirow{2}{*}{ CV (\%) } \\
\hline & & \multicolumn{2}{|l|}{ V1 } & \multicolumn{2}{|l|}{ V2 } & \multicolumn{3}{|c|}{ V3 } & & \\
\hline \multirow[t]{3}{*}{ Disco } & $\mathrm{H} 1$ & $0,0143 \mathrm{a}$ & $\mathrm{A}$ & $0,0184 \mathrm{a}$ & $\mathrm{A}$ & 0,0153 & $\mathrm{a}$ & $\mathrm{A}$ & $0,0160 \mathrm{a}$ & 22,9 \\
\hline & $\mathrm{H} 2$ & 0,0613 cde & $\mathrm{A}$ & $0,0684 \mathrm{~d}$ & A & 0,0673 & $\mathrm{c}$ & $\mathrm{A}$ & $0,0657 \mathrm{bc}$ & 7,2 \\
\hline & $\mathrm{H} 3$ & $0,0498 \mathrm{bc}$ & $\mathrm{A}$ & $0,0456 \mathrm{bc}$ & A & 0,0474 & $\mathrm{~b}$ & $\mathrm{~A}$ & $0,0476 \mathrm{~b}$ & 6,8 \\
\hline \multirow{3}{*}{$\begin{array}{l}\text { Cincel } \\
\text { vibratorio }\end{array}$} & $\mathrm{H} 1$ & $0,0339 \mathrm{~b}$ & $\mathrm{~A}$ & $0,0277 \mathrm{ab}$ & A & 0,0282 & $\mathrm{a}$ & A & $0,0300 \mathrm{a}$ & 31,3 \\
\hline & $\mathrm{H} 2$ & 0,0726 de & A & $0,0696 \mathrm{~d}$ & A & 0,0623 & $\mathrm{bc}$ & A & $0,0682 \mathrm{c}$ & 19,5 \\
\hline & H3 & $0,0570 \mathrm{~cd}$ & A & $0,0516 \mathrm{~cd}$ & A & 0,0572 & $\mathrm{bc}$ & $\mathrm{A}$ & $0,0553 \mathrm{~b}$ & 18 \\
\hline \multirow{3}{*}{$\begin{array}{l}\text { Cincel } \\
\text { rígido }\end{array}$} & $\mathrm{H} 1$ & $0,1468 \mathrm{~g}$ & $\mathrm{~B}$ & $0,1194 \mathrm{f}$ & $\mathrm{A}$ & 0,1427 & $\mathrm{e}$ & $\mathrm{B}$ & $0,1363 \mathrm{e}$ & 17,4 \\
\hline & $\mathrm{H} 2$ & $0,0917 \mathrm{f}$ & A & $0,0985 \mathrm{e}$ & A & 0,1023 & $\mathrm{~d}$ & $\mathrm{~A}$ & $0,0975 \mathrm{~d}$ & 24,1 \\
\hline & H3 & 0,0770 ef & A & $0,0693 \mathrm{~d}$ & A & 0,0704 & $\mathrm{c}$ & A & $0,0723 \mathrm{c}$ & 13,6 \\
\hline
\end{tabular}

V1: velocidad de 1,12 $\mathrm{m} \mathrm{s}^{-1}$; V2: velocidad de 1,45 $\mathrm{m} \mathrm{s}^{-1}$; V3: velocidad de 1,80 $\mathrm{m} \mathrm{s}^{-1}$; H1: contenido de agua de $0,05 \mathrm{~g} \mathrm{~g}^{-1}$; $\mathrm{H} 2$ : contenido de agua de $0,12 \mathrm{~g} \mathrm{~g} \mathrm{~g}^{-1}$; H3: contenido de agua de $0,15 \mathrm{~g} \mathrm{~g}^{-1}$; CV: coeficiente de variación.

Valores seguidos de letras minúsculas distintas en columnas o de letras mayúsculas en filas, indican diferencias significativas, según prueba de Duncan $(\mathrm{P}<0,05)$. 
Cuadro 3. Profundidad de trabajo (m) de los implementos, para diferentes contenidos de agua y velocidades de operación.

Table 3. Working depth (m) of the tillage tools, for different soil water contents and field speeds.

\begin{tabular}{|c|c|c|c|c|c|c|c|c|}
\hline \multirow{3}{*}{$\begin{array}{l}\text { Implemento } \\
\text { Disco }\end{array}$} & \multirow{3}{*}{$\begin{array}{c}\text { Humedad } \\
\mathrm{H} 1\end{array}$} & \multicolumn{5}{|c|}{ Velocidad de operación } & \multirow{2}{*}{ Media } & \multirow{3}{*}{$\begin{array}{r}\text { CV (\%) } \\
63,3\end{array}$} \\
\hline & & \multicolumn{2}{|c|}{ V1 } & \multicolumn{2}{|l|}{ V2 } & V3 & & \\
\hline & & $0,134 \mathrm{a}$ & A & $0,080 \mathrm{a}$ & A & 0,067 a $\mathrm{A}$ & 0,094 a & \\
\hline & $\mathrm{H} 2$ & $0,184 \mathrm{c}$ & A & $0,189 \mathrm{c}$ & $\mathrm{A}$ & 0,192 c A & $0,188 \mathrm{c}$ & 2,9 \\
\hline & $\mathrm{H} 3$ & $0,170 \mathrm{bc}$ & $\mathrm{B}$ & $0,157 \mathrm{~b}$ & $\mathrm{~A}$ & $0,163 \mathrm{~b} \quad \mathrm{AB}$ & $0,163 \mathrm{bc}$ & 4,5 \\
\hline \multirow{3}{*}{$\begin{array}{l}\text { Cincel } \\
\text { vibratorio }\end{array}$} & $\mathrm{H} 1$ & $0,152 \mathrm{ab}$ & A & $0,152 \mathrm{~b}$ & $\mathrm{~A}$ & $0,153 \mathrm{~b} \quad \mathrm{~A}$ & $0,152 \mathrm{~b}$ & 9,6 \\
\hline & $\mathrm{H} 2$ & $0,285 \mathrm{de}$ & A & $0,276 \mathrm{e}$ & $\mathrm{A}$ & 0,268 e $\mathrm{A}$ & $0,276 \mathrm{e}$ & 7,7 \\
\hline & $\mathrm{H} 3$ & 0,303 ef & A & 0,295 ef & A & $0,303 \mathrm{f} \quad \mathrm{A}$ & $0,300 \mathrm{f}$ & 6,6 \\
\hline \multirow{3}{*}{$\begin{array}{l}\text { Cincel } \\
\text { rígido }\end{array}$} & $\mathrm{H} 1$ & $0,325 \mathrm{f}$ & $\mathrm{B}$ & 0,289 ef & A & $0,295 \mathrm{f} \quad \mathrm{A}$ & $0,303 \mathrm{f}$ & 8,1 \\
\hline & $\mathrm{H} 2$ & $0,325 \mathrm{f}$ & A & $0,313 \mathrm{f}$ & $\mathrm{A}$ & $0,326 \mathrm{~g} \quad \mathrm{~A}$ & $0,321 \mathrm{~g}$ & 7,2 \\
\hline & $\mathrm{H} 3$ & $0,261 \mathrm{~d}$ & $\mathrm{~B}$ & $0,252 \mathrm{~d}$ & $\mathrm{AB}$ & $0,248 \mathrm{~d} \quad \mathrm{~A}$ & $0,254 \mathrm{~d}$ & 5,9 \\
\hline
\end{tabular}

V1: velocidad de $1,12 \mathrm{~m} \mathrm{~s}^{-1}$; V2: velocidad de 1,45 $\mathrm{m} \mathrm{s}^{-1} ; \mathrm{V} 3$ : velocidad de $1,80 \mathrm{~m} \mathrm{~s}^{-1} ; \mathrm{H} 1$ : contenido de agua de $0,05 \mathrm{~g} \mathrm{~g}^{-1} ; \mathrm{H} 2$ : contenido de agua de $0,12 \mathrm{~g} \mathrm{~g}^{-1} ; \mathrm{H} 3$ : contenido de agua de $0,15 \mathrm{~g} \mathrm{~g}^{-1} ; \mathrm{CV}$ : coeficiente de variación.

Valores seguidos de letras minúsculas distintas en columnas o de letras mayúsculas en filas, indican diferencias significativas, según prueba de Duncan $(\mathrm{P}<0,05)$.

Cuadro 4. Ancho de trabajo (m) de los implementos, para diferentes contenidos de agua y velocidades de operación. Table 4. Working width $(\mathrm{m})$ of tillage tools, for different soil water contents and field speeds.

\begin{tabular}{|c|c|c|c|c|c|c|c|c|c|}
\hline \multirow{2}{*}{ Implemento } & \multirow{2}{*}{ Humedad } & \multicolumn{6}{|c|}{ Velocidad de operación } & \multirow{2}{*}{ Media } & \multirow{2}{*}{ CV $(\%)$} \\
\hline & & \multicolumn{2}{|c|}{ V1 } & \multicolumn{3}{|c|}{ V2 } & V3 & & \\
\hline \multirow[t]{3}{*}{ Disco } & H1 & $0,417 \mathrm{a}$ & A & 0,433 & $\mathrm{a}$ & A & 0,417 a $\mathrm{A}$ & $0,422 \mathrm{a}$ & 10,5 \\
\hline & $\mathrm{H} 2$ & $0,633 \mathrm{bcd}$ & A & 0,650 & c & A & $0,600 \mathrm{bc} A$ & $0,628 \mathrm{~cd}$ & 8,1 \\
\hline & $\mathrm{H} 3$ & $0,550 \mathrm{bc}$ & $\mathrm{A}$ & 0,533 & $\mathrm{~b}$ & A & $0,567 \mathrm{bc} A$ & $0,550 \mathrm{bc}$ & 4,6 \\
\hline \multirow{3}{*}{$\begin{array}{l}\text { Cincel } \\
\text { vibratorio }\end{array}$} & $\mathrm{H} 1$ & $0,575 \mathrm{bc}$ & $\mathrm{B}$ & 0,463 & $\mathrm{ab}$ & A & $0,508 \mathrm{ab} A B$ & $0,515 \mathrm{~b}$ & 22,8 \\
\hline & $\mathrm{H} 2$ & $0,529 \mathrm{abc}$ & A & 0,500 & $\mathrm{ab}$ & A & $0,496 \mathrm{ab} A$ & $0,508 \mathrm{~b}$ & 8,3 \\
\hline & $\mathrm{H} 3$ & $0,517 \mathrm{ab}$ & $\mathrm{A}$ & 0,488 & $\mathrm{ab}$ & A & $0,504 \mathrm{ab} \mathrm{A}$ & $0,503 \mathrm{ab}$ & 12,6 \\
\hline \multirow{3}{*}{$\begin{array}{l}\text { Cincel } \\
\text { rígido }\end{array}$} & $\mathrm{H} 1$ & $0,967 \mathrm{e}$ & $\mathrm{A}$ & 0,946 & $\mathrm{~d}$ & A & 0,958 e $\mathrm{A}$ & $0,957 \mathrm{f}$ & 12,7 \\
\hline & $\mathrm{H} 2$ & $0,646 \mathrm{~cd}$ & A & 0,675 & $\mathrm{c}$ & A & $0,625 \mathrm{c} \quad \mathrm{A}$ & $0,649 \mathrm{~d}$ & 13,6 \\
\hline & H3 & $0,725 \mathrm{~d}$ & $\mathrm{AB}$ & 0,667 & c & A & $0,750 \mathrm{~d} \quad B$ & $0,714 \mathrm{e}$ & 12 \\
\hline
\end{tabular}

V1: velocidad de 1,12 $\mathrm{m} \mathrm{s}^{-1}$; V2: velocidad de 1,45 $\mathrm{m} \mathrm{s}^{-1} ; \mathrm{V} 3$ : velocidad de 1,80 $\mathrm{m} \mathrm{s}^{-1}$; H1: contenido de agua de $0,05 \mathrm{~g} \mathrm{~g}^{-1}$; H2: contenido de agua de $0,12 \mathrm{~g} \mathrm{~g}^{-1} ; \mathrm{H} 3$ : contenido de agua de $0,15 \mathrm{~g} \mathrm{~g}^{-1} ; \mathrm{CV}$ : coeficiente de variación.

Valores seguidos de letras minúsculas distintas en columnas o de letras mayúsculas en filas, indican diferencias significativas, según prueba de Duncan $(\mathrm{P}<0,05)$.

presentó para $\mathrm{H} 2$. El arado de disco también presentó un mayor ancho de trabajo para $\mathrm{H} 2 \mathrm{y}$, al igual que la profundidad de trabajo, este parámetro se ve afectado en condiciones de bajo contenido de humedad, donde se presentó el menor ancho de trabajo. El ancho de trabajo del cincel vibratorio no se ve afectado por el contenido de agua ni por la velocidad de operación. De igual manera, la velocidad de operación no afecta significativamente el ancho de trabajo en los casos del disco y del cincel rígi- do. Este parámetro está directamente relacionado con la distancia lateral de falla o cizallamiento en el suelo (McKyes, 1985) y sirve para determinar la distancia adecuada entre una unidad y otra, lo cual indica que el cincel vibratorio puede mantener una distancia fija, independiente del contenido de humedad. En el caso del arado de cincel rígido es necesario variar dicha distancia, la cual debe estar entre 1,4 $\pm 0,25$ veces la profundidad de trabajo (Godwin et al., 1984). 
Cuadro 5. Resistencia específica $\left(\mathrm{kN} \mathrm{m}^{-2}\right)$ de los implementos, para diferentes contenidos de agua y velocidades de operación.

Table 5. Specific draught $\left(\mathrm{kN} \mathrm{m}^{-2}\right)$ of tillage tools, for different soil water contents and field speeds.

\begin{tabular}{|c|c|c|c|c|c|c|c|c|c|c|c|c|c|}
\hline \multirow{3}{*}{$\begin{array}{l}\text { Implemento } \\
\text { Disco }\end{array}$} & \multirow{3}{*}{$\begin{array}{c}\text { Humedad } \\
\text { H1 }\end{array}$} & \multicolumn{9}{|c|}{ Velocidad de operación } & \multirow{2}{*}{\multicolumn{2}{|c|}{ Media }} & \multirow{3}{*}{$\begin{array}{r}\text { CV (\%) } \\
27,70\end{array}$} \\
\hline & & \multicolumn{3}{|c|}{ V1 } & \multicolumn{3}{|c|}{$\mathrm{V} 2$} & \multicolumn{3}{|c|}{ V3 } & & & \\
\hline & & 38,10 & $\mathrm{~d}$ & $\mathrm{~A}$ & 27,62 & $\mathrm{c}$ & $\mathrm{A}$ & 35,25 & $\mathrm{~d}$ & $\mathrm{~A}$ & 33,65 & $\mathrm{c}$ & \\
\hline & $\mathrm{H} 2$ & 9,35 & $\mathrm{a}$ & A & 8,39 & $\mathrm{a}$ & $\mathrm{A}$ & 8,51 & $\mathrm{a}$ & A & 8,75 & $\mathrm{a}$ & 7,50 \\
\hline & $\mathrm{H} 3$ & 20,56 & $\mathrm{~b}$ & $\mathrm{~A}$ & 22,35 & $\mathrm{bc}$ & $\mathrm{A}$ & 21,45 & $\mathrm{~b}$ & $\mathrm{~A}$ & 21,46 & $\mathrm{~b}$ & 6,50 \\
\hline \multirow{3}{*}{$\begin{array}{l}\text { Cincel } \\
\text { vibratorio }\end{array}$} & H1 & 36,58 & d & A & 46,69 & $\mathrm{~d}$ & A & 41,46 & $\mathrm{e}$ & A & 41,58 & d & 30,60 \\
\hline & $\mathrm{H} 2$ & 11,05 & $\mathrm{a}$ & A & 11,18 & $\mathrm{a}$ & $\mathrm{A}$ & 12,51 & $\mathrm{a}$ & $\mathrm{A}$ & 11,58 & $\mathrm{a}$ & 19,70 \\
\hline & $\mathrm{H} 3$ & 28,91 & $\mathrm{c}$ & A & 31,47 & $\mathrm{c}$ & $\mathrm{A}$ & 28,83 & $\mathrm{c}$ & A & 29,74 & $\mathrm{c}$ & 19,10 \\
\hline \multirow{3}{*}{$\begin{array}{l}\text { Cincel } \\
\text { rígido }\end{array}$} & $\mathrm{H} 1$ & 11,88 & $\mathrm{a}$ & $\mathrm{A}$ & 14,44 & $a b$ & $\mathrm{~A}$ & 12,38 & $\mathrm{a}$ & $\mathrm{A}$ & 12,90 & $\mathrm{a}$ & 20,00 \\
\hline & $\mathrm{H} 2$ & 12,45 & $\mathrm{a}$ & A & 11,76 & $\mathrm{a}$ & A & 12,34 & & A & 12,18 & $\mathrm{a}$ & 25,20 \\
\hline & $\mathrm{H} 3$ & 21,77 & $\mathrm{~b}$ & A & 24,40 & $\mathrm{c}$ & A & 23,78 & $\mathrm{bc}$ & A & 23,32 & $\mathrm{~b}$ & 14,40 \\
\hline
\end{tabular}

V1: velocidad de 1,12 $\mathrm{m} \mathrm{s}^{-1}$; V2: velocidad de 1,45 $\mathrm{m} \mathrm{s}^{-1}$; V3: velocidad de 1,80 $\mathrm{m} \mathrm{s}^{-1} ; \mathrm{H} 1$ : contenido de agua de $0,05 \mathrm{~g} \mathrm{~g}^{-1} ; \mathrm{H} 2$ : contenido de agua de $0,12 \mathrm{~g} \mathrm{~g}^{-1} ; \mathrm{H} 3$ : contenido de agua de $0,15 \mathrm{~g} \mathrm{~g}^{-1} ; \mathrm{CV}$ : coeficiente de variación.

Valores seguidos de letras minúsculas distintas en columnas o de letras mayúsculas en filas, indican diferencias significativas, según prueba de Duncan $(\mathrm{P}<0,05)$.

Resistencia específica. La resistencia específica para todos los tratamientos y condiciones de trabajo se presentan en el Cuadro 5. Con H1 la resistencia específica es alta para el disco y el cincel vibratorio, requiriéndose por tanto de mayor fuerza para movilizar una misma cantidad de suelo, comparado con los otros contenidos de humedad. Se observó que $\mathrm{H} 2$ es una condición adecuada para laborar el suelo, ya que presenta los menores valores de resistencia específica en los tres tipos de implementos, lo cual favorece un menor requerimiento de potencia y un menor consumo de combustible. En el caso del cincel rígido, la mayor resistencia específica se presentó con $\mathrm{H} 3$, deduciéndose que con contenidos altos de agua del suelo este implemento no es adecuado. Comparando los resultados encontrados en el presente trabajo para el cincel rígido, con los reportados por Arvidsson et al. (2004) y Camacho y Magalhães (2004), en suelos de texturas similares se observa que a mayor profundidad de trabajo, la resistencia específica disminuye, como también es reportado por Kasisira y Du Plessis (2006). De igual forma que para los otros parámetros evaluados, la velocidad de operación no incide significativamente en la resistencia específica, situación reportada por Rahman y Chen (2001) y por Sahu y Raheman (2006), quienes encontraron que la profundidad de trabajo presenta una mayor influencia sobre la resistencia específica.

\section{CONCLUSIONES}

El contenido de agua del suelo afecta los requerimientos de fuerza de tracción, el área de suelo disturbada, la profundidad de trabajo, el ancho de trabajo y la resistencia específica para todos los implementos estudiados.

El arado de discos y el cincel vibratorio son más sensibles para la realización de labores con bajos contenidos de agua del suelo, afectando principalmente la profundidad de trabajo, que a su vez influye significativamente en el área de suelo disturbado y en la resistencia específica.

La velocidad de operación no incidió significativamente en el área de suelo disturbado, la profundidad de trabajo, el ancho de trabajo ni en la resistencia específica.

Un contenido de agua intermedio se presenta como la condición adecuada para laborar el suelo, donde la resistencia específica es menor, lo cual se traduce en un menor requerimiento de potencia y baja demanda de energía, con una mayor área de suelo disturbada. 


\section{LITERATURA CITADA}

Aluko, O.B., and D.A. Seig. 2000. An experimental investigation of the characteristics of and conditions for brittle fracture in two-dimensional soil cutting. Soil Tillage Res. 57:143-157.

Arvidsson, J., T. Keller, and K. Gustafsson. 2004. Specific draught for mouldboard plough, chisel plough and disc harrow at different water contents. Soil Tillage Res. 79:221-231.

Camacho, J.H., y P.S.G. Magalhães. 2004. Estudio de parámetros operacionales de discos dobles y cinceles parabólicos para prácticas culturales en áreas con residuos vegetales. Agric. Téc. (Chile) 64:58-65.

Chaudhuri, D. 2001. Performance evaluation of various types of furrow openers on seed drills. A review. J. Agric. Eng. Res. 79:125-137.

Coelho, J.L.D. 1998. Avaliação de elementos sulcadores para semeadora-adubadoras utilizadas em sistemas conservacionistas de manejo do solo. $71 \mathrm{p}$. Tesis Maestría Ingeniería Agrícola. Universidade Estadual de Campinas, Faculdade de Engenharia Agrícola, Campinas, São Paulo, Brasil.

De Toro, A., and J. Arvidsson. 2003. Influence of spring preparation date and soil water content on seedbed physical conditions of a clay soil in Sweden. Soil Tillage Res. 70:141-151.

Gill, W.R., A.C Bailey, and C.A. Reaves. 1982. Harrow disk curvature. Influence on soil penetration. Trans. ASAE 25:1180-1180.

Godwin, R.J., D.A. Seig, and M. Allott. 1987. Soil failure and force prediction for soil engaging disc. Soil Use Manage. 3:106-114.

Godwin, R.J., G. Spoor, and M.S. Soomro. 1984. The effect of tine arrangement on soil forces and disturbance. J. Agric. Eng. Res. 30:47-56.
Kasisira L.L., y H.L.M Du Plessis. 2006. Energy optimization for subsoilers in tandem in a sandy clay loam soil. Soil Tillage Res. 86:185-198.

Lanças, K.P., e S.H. Benez. 1988. Influência da velocidade de subsolagem no consumo de combustível e na área mobilizada do solo. Energ. agric. (Botucatu) 3:25-30.

Magalhães, P.S.G., e W.R. Souza. 1990. Subsolador: Influência de parâmetros geométricos no mecanismo de forças. 2:1033. XIX Congresso Brasileiro de Engenharia Agrícola, Piracicaba, São Paulo, Brasil, Julio de 1990. Sociedad Brasilera de Ingenieria Agrícola, Jaboticabal, Sao Paulo, Brasil.

McKyes, E. 1985. Soil cutting and tillage. 217 p. Elsevier, Amsterdam, The Netherlands.

Mello, R.C., e P.S.G. Magalhães. 1995. Desempenho operacional de um arado de discos. Bragantia (Campinas) 54:447-454.

Mouazen, A.M., and H. Ramon. 2002. A numericalstatistical hybrid modeling scheme for evaluation of draught requirements of a subsoiler cutting a sandy loam soil, as affected by moisture content, bulk density and depth. Soil Tillage Res. 63:155-165.

Rahman, S., and Y. Chen. 2001. Laboratory investigation of cutting forces and soil disturbance resulting from different manure incorporation tools in a loamy sand soil. Soil Tillage Res. 58:19-29.

Sahu, R.K., and H. Raheman. 2006. Draught prediction of agricultural implements using reference tillage tools in sandy clay loam soil. Biosyst. Eng. 94:275-284.

SPSS. 2004. Statistical package for social sciences. Version 12.0. 2004. SPSS Inc. Chicago, Illinois, USA. 